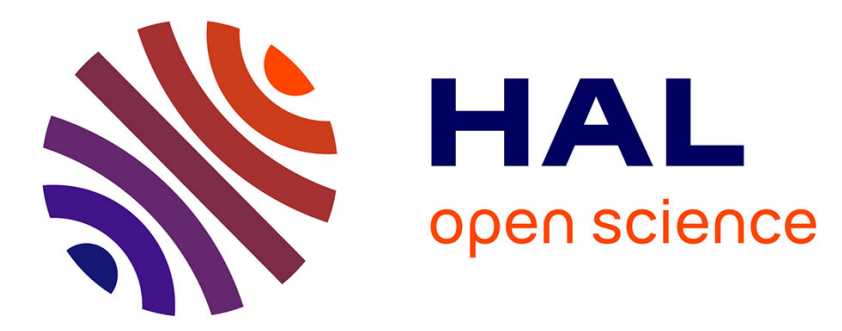

\title{
Assemblage par soudage diffusion de deux superalliages. Aspects diffusionnels et microstructuraux au niveau du joint
}

Yves Bienvenu, M.L. Dupont, F. Schwartz, J. Garcia

\section{- To cite this version:}

Yves Bienvenu, M.L. Dupont, F. Schwartz, J. Garcia. Assemblage par soudage diffusion de deux superalliages. Aspects diffusionnels et microstructuraux au niveau du joint. Journal de Physique IV Proceedings, 1994, 04 (C3), pp.C3-117-C3-122. 10.1051/jp4:1994316 . jpa-00252513

\section{HAL Id: jpa-00252513 https://hal.science/jpa-00252513}

Submitted on 1 Jan 1994

HAL is a multi-disciplinary open access archive for the deposit and dissemination of scientific research documents, whether they are published or not. The documents may come from teaching and research institutions in France or abroad, or from public or private research centers.
L'archive ouverte pluridisciplinaire $\mathbf{H A L}$, est destinée au dépôt et à la diffusion de documents scientifiques de niveau recherche, publiés ou non, émanant des établissements d'enseignement et de recherche français ou étrangers, des laboratoires publics ou privés. 


\title{
Assemblage par soudage diffusion de deux superalliages. Aspects diffusionnels et microstructuraux au niveau du joint
}

\author{
Y. BIENVENU, M.L. DUPONT ${ }^{(1)}$, F. SCHWARTZ* et J.C. GARCIA** \\ Centre des Matériaux de l'Ecole des Mines de Paris, BP. 87, 91003 Evry cedex, France \\ * SNECMA, Gennevilliers, France \\ ${ }^{* *}$ TURBOMECA, Tarnos, France
}

\begin{abstract}
Diffusion bonding is the prefered technique to join modern nickel based superalloys such as an aero-engine disk type material (processed by powder metallurgy to resist low cycle fatigue at moderate temperature) and a large grain cast or directionally solidified material resisting to creep. The microstructure in the vicinity of the joint is affected by diffusion, especially the $\gamma^{\prime}$ phase. In turn mechanical properties are affected by the transitions from one structure to the other.
\end{abstract}

\section{INTRODUCTION}

La plage de fonctionnement température-contrainte des composants chauds de turbomachines aéronautiques évolue, rendant plus délicate la réalisation de ces composants en un seul matériau à microstructure uniforme. On a intérêt à associer un matériau à gros grain (aubes et périphérie de disque) à un matériau à grain fin (centre du disque plus froid mais soumis à un niveau de contrainte proche de la limite d'élasticité). Les assemblages rigides aubes/disque se justifient aussi en termes d'aérodynamique et de vibrations. Les deux applications de l'assemblage métallurgique les plus fréquemment mentionnées sont illustrées sur la figure 1.

L'assemblage de deux superalliages peut permettre la réalisation de roue monobloc (aubes obtenues par solidification dirigée + disque obtenu par métallurgie des poudres) remplaçant les fixations mécaniques actuelles en pied de sapin. Une autre application est la fabrication de disques à structure hybride avec, dans la partie jante, un alliage résistant au fluage (température $>650^{\circ} \mathrm{C}$ et contraintes $>500 \mathrm{MPa}$ ) et dans la partie alésage un alliage résistant à la fatigue oligocyclique $\left(2\right.$ à 3 cycles par vol, $400^{\circ} \mathrm{C}$ et contraintes $>900 \mathrm{MPa}$ ) (figure 1).

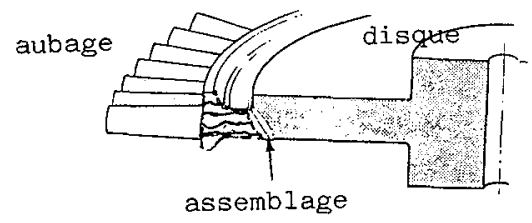

a)

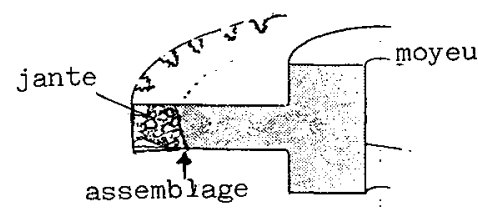

b)

Figure 1 Représentation schématique de deux applications du soudage par diffusion de superalliages à base de nickel a) roue monobloc b) disque hybride.

Pour la majorité des superalliages modernes à base de nickel ,le soudage par fusion est exclus; il faut avoir recours au soudage par diffusion, au soudage par friction [1,2].ou au brasage-diffusion.

(1) Actuellement : Université Paris-Sud, Orsay 
Le soudage par diffusion a pour avantages l'absence de zone affectée thermiquement, la possibilité d'assembler des matériaux de microstructures très différentes et une déformation plastique limitée des deux composants. Parmi les inconvénients les plus importants, figurent une productivité faible ainsi qu'un coût d'investissement assez élevé.

Trois modes d'assemblage sont étudiés par les auteurs depuis quelques années [3,4] : en laboratoire et à l'échelle pré-développement : compaction uniaxiale à chaud (CUC), compaction isostatique à chaud (CIC) et cofilage à chaud. CUC et CIC peuvent conduire à des assemblages près des cotes. Le cofilage à chaud conduit à des preformes de forgeage et permet de combiner densification et assemblage. Il offre des perspectives de coût unitaire intéressantes pour l'élaboration de disque hybride (figure lb).

\section{MATERIAUX ET PROCEDES}

Dans toutes nos études, l'Astroloy NK17CDAT, prototype des matériaux pour disques chauds est utilisé. C'est un alliage à grain fin $(7 \mu \mathrm{m}$ à $30 \mu \mathrm{m})$ obtenu par métallurgie des poudres.

Différents alliages ayant un grain grossier ont été associés à l'Astroloy. Ceux-ci diffèrent par leurs compositions (tableau 1) et leur structure. Waspaloy(NC20K13DTA) et IN100 (NK15CATD) sont équiaxes alors que le DS247 (NKW9CATaHf) est colonnaire, ce dernier résiste le mieux au fluage.

Tableau 1. Composition et solvus $\gamma^{\prime}$ des superalliages utilisés pour les assemblages (masse.\%, Ni=reste)

\begin{tabular}{|c|c|c|c|c|c|c|c|c|}
\hline $\begin{array}{c}\text { Eléments } \\
\text { Alliages }\end{array}$ & Co & $\mathrm{Cr}$ & $\mathrm{Al}$ & $\mathrm{Ti}$ & $\mathrm{Mo}$ & W, Ta,Hf & $\mathrm{C}$ & $\begin{array}{c}\gamma^{\prime} \text { Solvus } \\
\mathrm{C}\end{array}$ \\
\hline Astroloy & 16.9 & 14.8 & 3.87 & 3.45 & 5.1 & - & 0.015 & 1135 \\
\hline IN 100 & 15.4 & 10.0 & 5.4 & 4.11 & 3.0 & - & 0.14 & 1200 \\
\hline DS 247 & 10 & 8 & 5.5 & 1 & 0.6 & $\begin{array}{c}10 . \mathrm{W} \\
\text { 3.0Ta } \\
\text { 1.5Hf }\end{array}$ & 0.06 & 1235 \\
\hline Waspaloy & 13.5 & 20 & 1.4 & 3 & 4 & & 0.07 & 1020 \\
\hline
\end{tabular}

La température de solvus $\gamma$ est une température caractéristique au-dessus de laquelle les précipités $\gamma^{\prime}$, cohérents avec la matrice $\gamma$, cfe se dissolvent laissant ainsi grossir le grain. Ces superalliages doivent leurs bonnes caractéristiques mécaniques à ces précipités $\gamma^{\prime}$, de taille inférieure à $2 \mu \mathrm{m}$ et de fraction volumique approximative égale à $20 \%$ pour Waspaloy, $45 \%$ pour Astroloy, $70 \%$ pour DS247 ainsi qu'aux éléments ( $\mathrm{Cr}, \mathrm{W}, \mathrm{Mo}, \mathrm{Ta})$ en solution solide dans $\gamma$.

Plusieurs conditions d'assemblage sont étudiées :

- C.U.C. : cylindres sous vide secondaire, $\emptyset 20 \mathrm{ou} 10 \mathrm{~mm}, \mathrm{~h} \sim 25 \mathrm{~mm}$ mis bout à bout sous la presse à chaud de $20 \mathrm{t}$, les deux matériaux sont sous forme massive,

- C.I.C. : la compaction se fait sous conteneur dans une presse de laboratoire. Deux échelles de dimension conteneurs $\emptyset 15, \mathrm{~h} 50 \mathrm{~mm}$ ou conteneurs $\varnothing 80, \mathrm{~h} \sim 180$ sont abordées successivement . Il a été possible d'assembler deux superalliages sous forme de poudres en pré-frittant légèrement une poudre d'Astroloy sous vide à $1100^{\circ} \mathrm{C}$ avant de la placer au centre d'un conteneur rempli de poudre de Waspaloy mais généralement l'un des deux ou les deux matériaux sont denses,

- le cofilage est réalisé comme la CIC sous conteneur en presse à chaud de $1000 \mathrm{t}$. Avant extrusion, on procedde à un pressage uniaxial à chaud pour réduire la porosité de la poudre de $35 \%$ à $20 \%$, le taux d'extrusion est de l'ordre de 5. CIC et cofilage sont suivis d'un forgeage isotherme vers $1100^{\circ} \mathrm{C}$. 


\section{SYNTHESE DES PRINCIPAUX RESULTATS ET DISCUSSIONS}

\subsection{Porosités localisées à l'interface}

Des trois procédés étudiés, l'assemblage par codéformation est le plus sûr pour l'élimination des porosités interfaciales, à cause du niveau important de déformation imposé lors du filage à chaud.

Pour le pressage uniaxial, et particulièrement pour la combinaison Astroloy/DS247, il est nécessaire de dépasser la température de solvus de l'alliage le moins réfractaire (Astroloy). Pour une durée de maintien donnée $(0.5$ à $2 \mathrm{~h})$, on peut dégager une relation température-pression qui est nécessairement étroite car on exige l'élimination des porosités tout en limitant la déformation à 0.01 par exemple pour réaliser des pièces aux cotes. Cette relation est parallèle à l'évolution de la limite d'elasticité.

Pour le pressage isostatique à chaud, les seuls cas d'observation de porosité interfaciale sont ceux alliant faible pression et faible température, par exemple $60 \mathrm{MPa}-1100^{\circ} \mathrm{C}$ pour le couple Astroloy-IN100.

L'élimination des porosités ne signifie pas nécessairement qu'il y ait véritablement liaison. Pour réaliser des assemblages Astroloy-DS247 à basse température $\left(1120^{\circ} \mathrm{C}\right)$ présentant sous sollicitation en traction une rupture non localisée à l'interface, il est nécessaire d'interposer un insert en métal, un film de nickel par exemple, dont la limite d'élasticité est inférieure à celle de l'Astroloy..

\subsection{Précipitations à l'interface (figure 2)}

Avec différents couples de superalliages dont l'un au moins est riche en titane, la formation de TiC à l'interface peut avoir lieu pendant l'assemblage. Ceci est lié à une coségrégation de Ti et $\mathrm{C}$ en surface [5,6] qui peut s'interpréter par une approche thermodynamique. Ceci est observé sous vide secondaire; mais, si la pression partielle d'oxygène lors de l'assemblage est suffisante, ce sont des oxydes de hafnium qui peuvent être observés - cas de l'assemblage Astroloy/DS247 par compaction isostatique à chaud -.

Lors du cofilage, le niveau de déformation est tel que l'on n'observe pas, comme en C.I.C., d'alignements rectilignes et après forgeage isotherme, on n'observe que de courts $(25 \mu \mathrm{m})$ alignements de carbures. En dehors de la déformation plastique, les mesures à prendre pour limiter la nuisance associée aux carbures sont d'ordre physico-chimique: c'est à dire qu'il convient d'éliminer une partie du titane et du carbone par un traitement en atmosphère fluorée préalable à l'assemblage $[3,4]$.
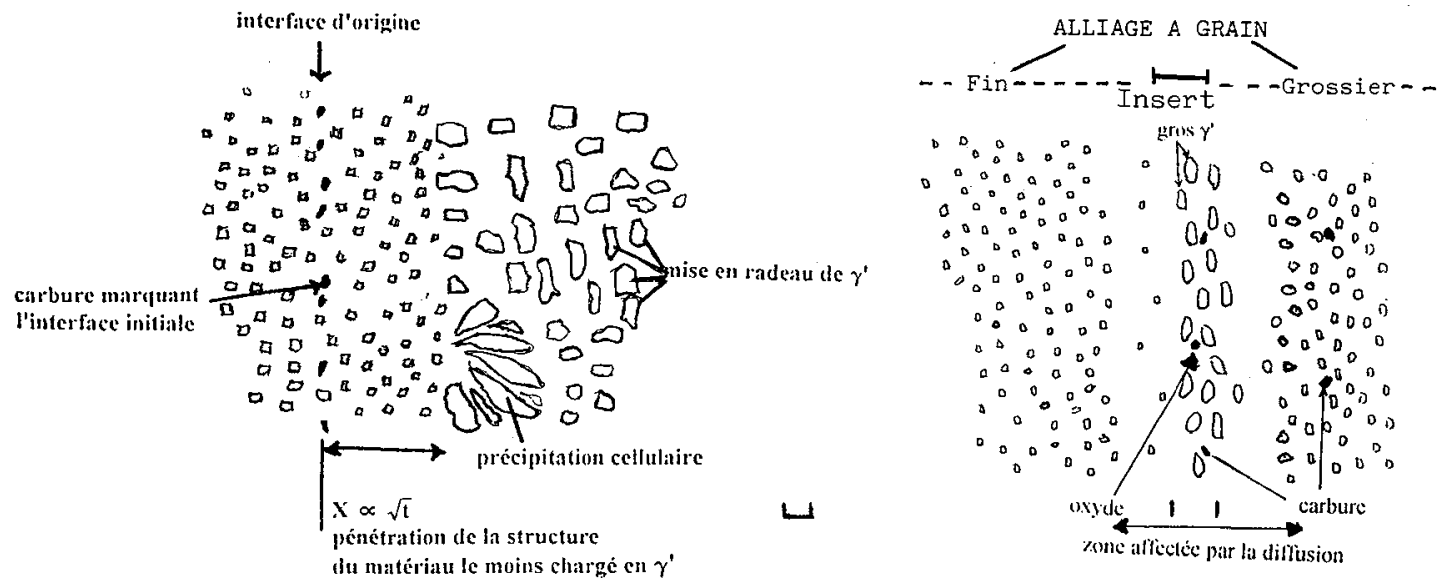

Figure 2 Représentation schématique des modifications microstructurales a) assemblage sans insert de nickel b) assemblage avec insert de nickel (symbole creux $=\gamma$, symboles pleins $=$ carbure) 


\subsection{Evolution de la précipitation gamma prime au voisinage de l'interface - aspects diffusionnels.}

L'assemblage est de préférence réalisé à une température intermédiaire entre les solvus $\gamma^{\prime}$ des deux alliages, ce qui, après refroidissement, se traduit par une précipitation $\gamma$ fine pour l'alliage le moins réfractaire et grossière pour le plus réfractaire. La transition entre les deux microstructures est abrupte bien que les profils de concentration des différents éléments dans la matrice $\gamma$ soient continus. La frontière se déplace en fonction du temps d'une distance $X$ par rapport à l'interface d'origine (repérée par les marqueurs que constituent les précipités de carbures ou d'oxydes) avec en première approximation :

$$
\mathrm{X}^{2}=\mathrm{H} \mathrm{t}
$$

Le problème de diffusion est complexe : système à 10-12 composants, existence d'un (ou de deux) front(s) de dissolution de précipités, processus qui constitue une source d'aluminium et de titane. De l'étude de l'équation d'équilibre des flux à cette frontière, on peut démontrer que $\mathrm{H}$ est de l'ordre de $\mathrm{D}$. Effectivement, les valeurs mesurées (qui se situent entre $10^{-11}$ et $10^{-10} \mathrm{~cm}^{2} / \mathrm{s}$ vers $1100^{\circ} \mathrm{C}$ ) pour différents couples [1], de la constante cinétique $\mathrm{H}$ sont proches de celles des coefficients de diffusion de l'aluminium dans la phase $\gamma$ du système $\mathrm{Ni}-\mathrm{Al}$ [7].

Dans le cas de l'assemblage de deux superalliages à base de nickel (Astroloy/DS247), avec un insert de nickel, on observe une zone blanche, (c'est à dire sans précipités $\gamma^{\prime}$ de taille supérieure à $0,1 \mu \mathrm{m}$ ) de part et d'autre de l'insert, celle développée du côté Astroloy étant plus importante (largeur jusqu'à $20 \mu \mathrm{m}$ ).

La résolution des équations de la diffusion peut être tentée pour l'aluminium, par exemple, dans le cas du couple Waspaloy-Astroloy. On aboutit à l'aide de solutions approchées [8] à $\mathrm{D}_{\mathrm{Al}} \sim 5 \times 10^{-10} \mathrm{~cm}^{2 / \mathrm{s}}$, valeur en bon accord avec celles données dans la littérature [7], et ce, dans le cas de profils de concentration déterminés à la microsonde (figure 3).

Lorsqu'un film de nickel est interposé, on observe une situation fort complexe au niveau de la précipitation $\gamma$ et du profil de concentration (figures $2 \mathrm{~b}-3 \mathrm{~b}$ ). L'aluminium et le titane diffusent plus vite dans le film de nickel que les éléments intervenant pour le durcissement en solution solide; ceci se traduit par un maximum local de la concentration d'aluminium au milieu du film de nickel entraînant une augmentation locale de la température de solvus (figure 3) et la précipitation grossière de $\gamma$ au mileu de l'insert. Ceci est plus marqué lorsque l'assemblage est réalisé à $1200^{\circ} \mathrm{C}$ plutôt qu'à $1120^{\circ} \mathrm{C}$ pour le couple Astroloy/DS247 et on aboutit alors à un paradoxe apparent : l'utilisation de températures plus élevées $\left(1200^{\circ} \mathrm{C}\right)$ conduit à une plus grande hétérogénéité de la précipitation $\gamma$ (figure $2 \mathrm{~b}$ ).

Les évolutions microstructurales de la précipitation $\gamma$ ne sont pas limitées aux seules dissolutions et reprécipitations par diffusion mais résultent aussi de réactions localisées qui sont illustrées schématiquement figure 2; il peut se produire :

- une précipitation cellulaire de $\gamma$ qui provient d'une dissolution de fins précipités $\gamma$ en avant du front et une reprécipitation en arrière sous forme de lamelles grossières d'allure eutectoïde. La force motrice de cette réaction est la réduction d'énergie interfaciale $\gamma-\gamma^{\prime}$ ainsi que la réduction de la densité des dislocations et donc de l'énergie associée La redistribution des éléments $\gamma$-gènes et $\gamma$-gènes sur la surface du front de dissolution cellulaire contrôle la cinétique de la précipitation cellulaire.

- dans certaines circonstances, une mise en radeau des gros précipités $\gamma$ (figure 2) affectant l'alliage le plus réfractaire des assemblages, c'est à dire l'IN100 ou le DS247. Ces circonstances sont : assemblage sous contrainte uniaxiale (CUC) et existence d'une différence sensible de paramètre cristallin entre $\gamma$ et $\gamma^{\prime}$. Cette mise en radeau n'est observée que sur une distance de l'interface qui correspond à la distance caractéristique de diffusion de l'aluminium (jusqu'à $50 \mu \mathrm{m}$ en pratique). 

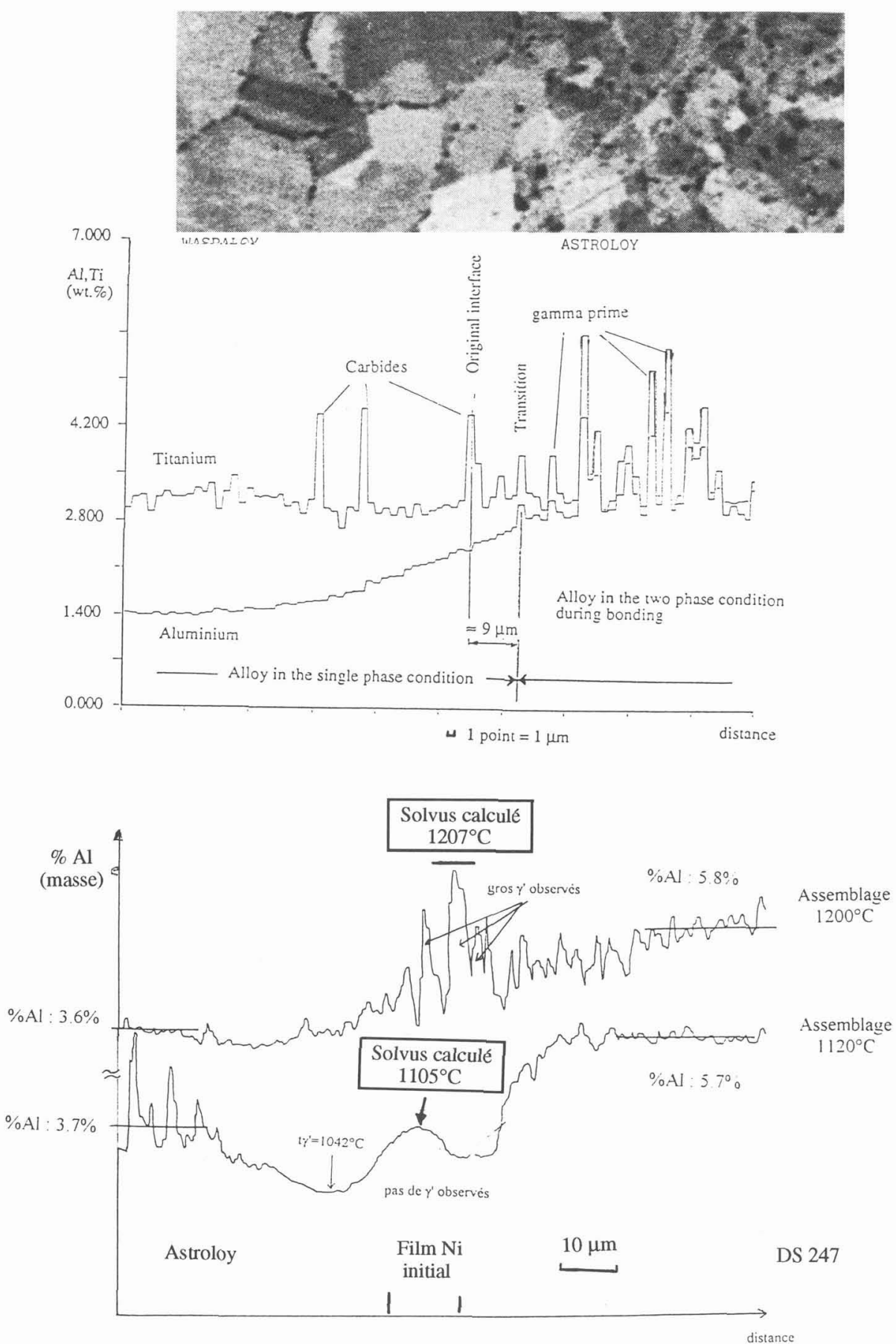

Figure 3 Profils de concentration des éléments $\mathrm{Al}$, Ti dans

a) une jonction Waspaloy / Astroloy sans insert à une température proche de $1100^{\circ} \mathrm{C}$. b) une jonction Astroloy / DS 247 avec film de nickel à $1120^{\circ} \mathrm{C}$ et $1200^{\circ} \mathrm{C}$. 


\section{PROPRIETES MECANIQUES}

Pour le couple Astroloy/Waspaloy, cofilé et forgé, les propriétés en traction (de l'ambiante à $650^{\circ} \mathrm{C}$ ) et en fluage (jusqu'à $750^{\circ} \mathrm{C}$ ) d'éprouvettes bi-matériaux sont proches de celle du Waspaloy (l'alliage le plus faible dans le domaine des sollicitations exploré). Lorsque l'interface est perpendiculaire à l'axe de sollicitation. Mais, lorsque l'interface est inclinée par rapport à cet axe, les propriétés sont plus proches de celles de l'alliage le plus résistant. Ce fait avait déjà été indiqué par Peterson lors de la caractérisation de bi-matériaux en fatigue [9] et mérite d'être étudié plus en détail.

Pour le couple DS247/Astroloy assemblé par CIC, il paraît actuellement indispensable d'utiliser un insert ductile (nickel) entre les deux superalliages. L'ensemble des propriétés mécaniques (traction-fluagefatigue) paraît alors satisfaisant pour les plus hautes températures d'assemblage, c'est à dire niveau de propriétés proches de l'alliage le plus faible, mais la présence de gros précipités gamma prime dans cet insert après diffusion poussée est un point négatif en ce qui concerne l'amorçage de fissures en fluage.

\section{CONCLUSIONS}

La diffusion contrôle la plupart des évolutions microstructurales au voisinage du joint réalisé par soudage-diffusion de superalliages à base de nickel et il est possible d'ajuster la profondeur de la transition microstructrurale ainsi que la taille des précipités par la durée du traitement et la taille des précipités gamma prime à l'interface par la température.

Le comportement mécanique des jonctions devrait pouvoir, à son tour, être optimisé par le contrôle des phénomènes diffusionnels.

\section{REFERENCES}

[1] Y. Bienvenu, B. Decaudin, T.J. Massart and F. Schwartz, Metallurgical aspects of solid joining of nickel base superalloys, B.A.B.S., $6^{\text {th }}$ International Conference, High Technology Joining, Strafford, 3-5 Sept. 1991.

[2] G.H. Gessinger, Powder Metallurgy of Superalloys, Butterworths, London (1984) 306.

[3] T.J. Massart, Thèse de Doctorat, Ecole Nationale Supérieure des Mines de Paris, 1987.

[4] T.J. Massart, A. Morrison, J.L. Koutny et Y. Bienvenu, Soudage par diffusion de superalliages à base de nickel : répercussions microstructurale, 4 èmes Journées Nationales du Soudage, Institut de Soudure et Sociétê des Ingénieurs Soudeurs, 14-16 mars 1989.

[5] M. Guttmann, Thèse Doctorat es Science, Université Paris XI, (1974).

[6] M. Dahlen, N.G. Ingesten et H. Fischmeister, Mod. Dev. in P.M., 14 (1981) 3.

[7] M.P. Janssen, Met. Trans. 4 (1973) p 1623.

[8] J. Philibert, Diffusion et transport de matière dans les solides, Edition de Physique, Les Ulis, France, 1986, 22.

[9] L.G. Perterson, D. Hrencecin, A.M. Ritter et N. Lewis, HIP diffusion bonding of P.M. alloys for composite land based bas turbine buckets, M.P.R., 41, 10, (1986). 